\title{
Smokeless tobacco use is associated with a substantial risk of oral cancers in India
}

\author{
Are there any health risks associated with smokeless tobacco?
}

\section{JA Critchley, B Unal. Health effects associated with smokeless tobacco: a systematic review. Thorax 2003; 58:435-443}

Data sources Medline, Embase, CINAHL and Dissertation Abstracts were searched, supplemented by screening reference lists, smokingrelated websites, and contacting experts.

Study selection Analytical observational studies of use of smokeless tobacco (ST; cohorts, case-control, cross-sectional studies) with a sample size of $\geq 500$ were included if they reported on one or more of the following outcomes: mortality from any cause, oral and pharyngeal cancers, other cancers, cardiovascular diseases, dental diseases, pregnancy outcomes or surgical outcomes.

Data extraction and synthesis Data extraction covered control of confounding, selection of cases and controls, sample size, clear definitions and measurements of the health outcome, and ST use. Selection, extraction and quality assessments were carried out by one or two independent reviewers.

Results Many of the studies lacked sufficient power to estimate precise risks, mainly because of the small number of ST users. Studies were often not designed to investigate ST use, and many also had major methodological limitations including poor control for cigarette smoking and imprecise measurements of exposure. Studies in India showed a substantial risk of oral or oropharyngeal cancers associated with chewing betel quid and tobacco. Studies from other regions and of other cancer types were not consistent. Few studies have adequately considered the non-cancer health effects of ST use.

Conclusions Chewing betel quid and tobacco is associated with a substantial risk of oral cancers in India. Most recent studies from the US and Scandinavia are not statistically significant, but moderate positive associations cannot be ruled out due to lack of power. Further rigorous studies with adequate sample sizes are required, especially for cardiovascular disease.

\section{Commentary}

With recent legislation to ban smoking in public places in Eire and Government debate within the UK, smoking has featured heavily in the media in recent months. The paper reviewed here is an important and timely reminder of the many different forms that tobacco-use can take and summarises the evidence related to the health risks which may be associated with ST.

The first striking thing about this paper is that, in direct contrast with the evidence relating to smoking tobacco, the review found that risks in Scandinavia were higher for individuals who had ceased ST-use than for current users. The authors suggest that this may be due to selection bias and anecdotal evidence of a reduction in carcinogenic tobacco-specific N-nitrosamines in ST. A recent paper confirms that this may well be a contributory factor in Sweden. ${ }^{1}$

Evidence linking the chewing of tobacco with dental caries was also reviewed and a suggestion made that sugar content may be influential. A recent paper based upon a survey of 13-15-year olds in India $^{2}$ gives an additional perspective as it reports the use of tobacco products as dentifrice in up to $68 \%$ of this age group. The paper did not report whether this replaced the use of fluoride toothpaste: if so, this could contribute to an increased prevalence in caries in those children compared with children who used fluoride toothpaste.

It appears that, outside of India, little research has focussed exclusively upon the health effects of ST. In systematically reviewing the literature, this paper identifies many of the important methodological considerations for future studies. This ought to ensure that future studies can withstand the rigours of critical appraisal and contribute towards our understanding of the risks of ST.

\section{Gail Topping \\ Dental Health Services Research Unit, University of Dundee, Dundee, Scotland, UK}

1. Osterdahl BG, Jansson C, Paccou A. Decreased levels of tobacco-specific $\mathrm{N}$-nitrosamines in moist snuff on the Swedish market. J Agric Food Chem 2004 52:5085-5088.

2. Sinha DN, Gupta PC, Pednekar MS. Use of tobacco products as dentifrice among adolescents in India: questionnaire study. BMJ 2004; 328:323-324.

Evidence-Based Dentistry (2004) 5, 79.

doi:10.1038/sj.ebd.6400280 